\title{
Locality of Queries and Transformations
}

\author{
(Invited Talk)
}

\author{
Marcelo Arenas * \\ Center for Web Research \& \\ Computer Science Department, Pontificia Universidad Católica de Chile, \\ Escuela de Ingeniería - DCC143, Casilla 306, Santiago 22, Chile. \\ marenas@ing.puc.cl
}

\begin{abstract}
Locality notions in logic say that the truth value of a formula can be determined locally, by looking at the isomorphism type of a small neighborhood of its free variables. Such notions have proved to be useful in many applications especially in computer science. They all, however, refer to isomorphism of neighborhoods, which most local logics cannot test. A more relaxed notion of locality says that the truth value of a formula is determined by what the logic itself can say about that small neighborhood. Or, since most logics are characterized by games, the truth value of a formula is determined by the type, with respect to a game, of that small neighborhood. Such game-based notions of locality can often be applied when traditional isomorphism-based locality cannot.

In the first part of this talk, we show some recent results on game-based notions of locality. We look at two, progressively more complicated locality notions, and we show that the overall picture is much more complicated than in the case of isomorphism-based notions of locality.

In the second part of this talk, we concentrate on the locality of transformations, rather than queries definable by formulas. In particular, we show how the game-based notions of locality can be used in data exchange settings to prove inexpressibility results.
\end{abstract}

* Partially supported by FONDECYT grant 1050701 and the Millennium Nucleus

Center for Web Research, Grant P04-067-F, Mideplan, Chile.

Please use the following format when citing this chapter:

Arenas, M., 2006, in International Federation for Information Processing, Volume 209, Fourth IFIP International Conference on Theoretical Computer Science-TCS 2006, eds. Navarro, G., Bertossi, L., Kohayakwa, Y., (Boston: Springer), p. 3. 